\title{
Anions in Space and in the Laboratory
}

\author{
Veronica M. Bierbaum \\ Department of Chemistry and Biochemistry \\ Center for Astrophysics and Space Astronomy \\ University of Colorado \\ Boulder, Colorado 80309-0215 \\ email: veronica.bierbaum@colorado.edu
}

\begin{abstract}
The astronomical detection of molecular anions has prompted our study of their chemical reactions with atomic species that are abundant in the interstellar medium. We have recently explored the chemistry of a variety of $\mathrm{C}_{x} \mathrm{~N}_{y}{ }^{-}$anions with hydrogen atoms and determined their reaction rate constants and products using the flowing afterglow-selected ion flow tube technique. Computational studies allow characterization of the structures of reactants and products, as well as the energetics along the reaction pathway. For anions containing one or two nitrogen atoms, reactions with hydrogen atoms are facile, and proceed primarily by associative detachment. In contrast, anions containing three nitrogen atoms are unreactive with hydrogen atoms due to reaction barriers and unfavorable thermodynamics.
\end{abstract}

Keywords. Negative ions, gas phase reactions, associative detachment, reaction rate constants, flowing afterglow-selected ion flow tube

\section{Introduction}

Ions are found throughout nature in many environments. They are abundant in the earth's oceans and atmosphere, as well as in lightning and flames; ions are critical in the biochemical reactions of life, and they have been detected in many space environments. Positive ions have been known to exist in the interstellar medium (ISM) since the discovery of $\mathrm{CH}^{+}$in 1941 (Douglas \& Herzberg 1941), but as recently as five years ago, scientists were debating whether negative ions could survive in these environments. Then in December 2006, McCarthy and co-workers reported the Laboratory and Astronomical Identification of the Negative Molecular Ion $C_{6} H^{-}$(McCarthy et al. 2006). A total of six anions have now been detected including three hydrogenated carbon chain anions, $\mathrm{C}_{4} \mathrm{H}^{-}, \mathrm{C}_{6} \mathrm{H}^{-}, \mathrm{C}_{8} \mathrm{H}^{-}$, and three cyano anions, $\mathrm{CN}^{-}, \mathrm{C}_{3} \mathrm{~N}^{-}, \mathrm{C}_{5} \mathrm{~N}^{-}$(McCarthy et al. 2006; Brünken et al. 2007; Cernicharo et al. 2007, 2008; Remijan et al. 2007; Thaddeus et al. 2007; Agúndez et al. 2010). These negative ions represent a large fraction of recently detected chemical species, and certainly many more are awaiting discovery.

Our research program has focused on the gas phase chemistry of ions with neutrals that are abundant in the ISM (Snow \& Bierbaum 2008), and the potential role of these reactions in the synthesis of interstellar molecules. This presentation will provide an overview of our studies of negative ion chemistry, and then describe our recent work on the nitrogen-containing carbanions, $\mathrm{C}_{x} \mathrm{~N}_{y}{ }^{-}$.

\section{Experimental}

We employ the versatile Flowing Afterglow-Selected Ion Flow Tube (FA-SIFT) Technique shown in Figure 1 (Van Doren et al. 1987). Ions are formed in a flowing afterglow source utilizing a variety of ionization methods, including dc discharge, electron impact, 


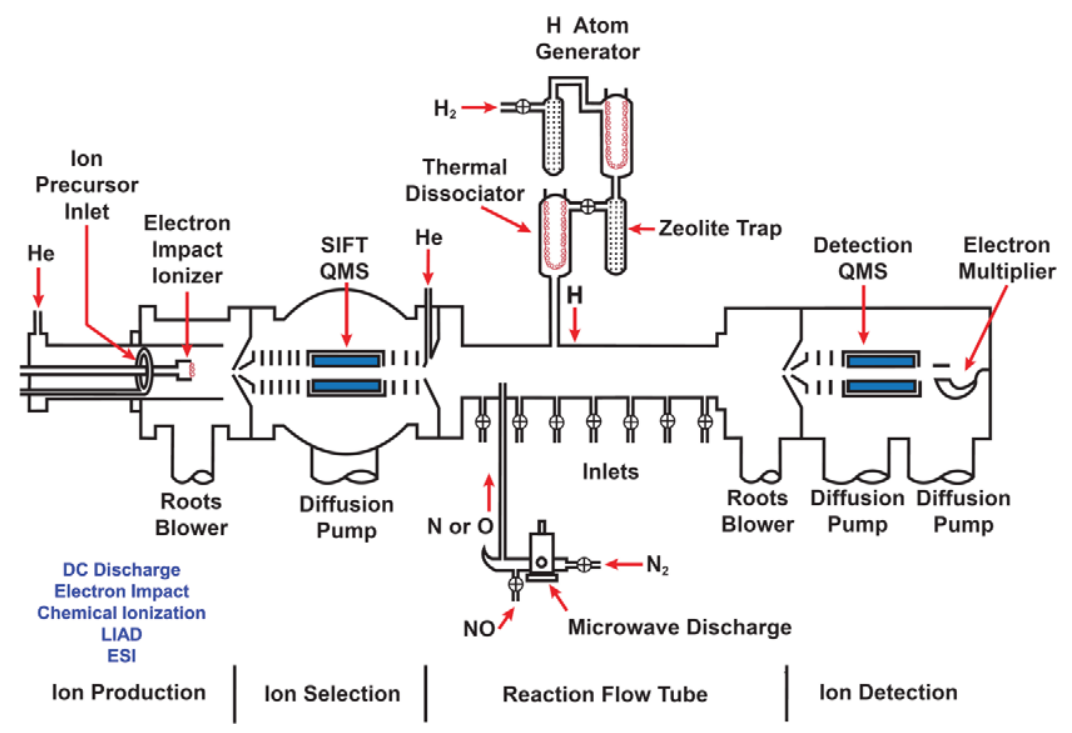

Figure 1. The Flowing Afterglow-Selected Ion Flow Tube (FA-SIFT).

and chemical ionization; these sources allow the formation and study of a wide array of ions. The desired reactant ion is mass-selected with a quadrupole mass filter, and injected through a venturi inlet into a stream of helium. The ions are thermalized by thousands of collisions with helium; stable molecular reactants are added through a manifold of inlets, and atomic reactants can also be introduced. Reaction occurs throughout the remainder of the flow tube; the reactant and product ions are monitored with a quadrupole mass filter, and kinetics are determined from the dependence of ion intensities on the flow rate of the neutral reactant. To generate $\mathrm{H}$-atoms, molecular hydrogen is dissociated thermally; hydrogen is passed through liquid nitrogen traps and over a hot filament. The flow of H-atoms is proportional to the voltage applied to the filament. The method produces a few percent dissociation and the hydrogen atom density is measured with calibration reactions. Nitrogen atoms are generated by passing molecular nitrogen through a microwave discharge; oxygen atoms are formed by addition of NO, which reacts with nitrogen atoms. The concentrations of $\mathrm{N}$-atoms and O-atoms are determined from the endpoint of the titration.

This method has several valuable features for studying gas phase ion chemistry; in particular, the ions are thermalized by many collisions with helium before reaction, so that the energy is well-defined; however, the energy can be varied by heating or cooling the flow tube, or applying a drift field; because distance and time are related in a flow tube, kinetic analysis, measurement of rate constants, is straightforward; there are high ion densities and good sensitivity - this has allowed coupling of the flowing afterglow with many other techniques over the years; perhaps the most valuable feature is the chemical versatility - a wide variety of ionic and neutral reactants can be formed and studied, including atomic reactants.

\section{Results}

Our studies of negative ions have included five categories: pure carbon chains $\left(\mathrm{C}_{x}{ }^{-}\right)$, hydrogenated carbon chains $\left(\mathrm{C}_{x} \mathrm{H}^{-}\right)$, organic anions $\left(\mathrm{H}_{w} \mathrm{C}_{x} \mathrm{~N}_{y} \mathrm{O}_{z}^{-}\right)$, hydride anion $\left(\mathrm{H}^{-}\right)$, and nitrogen-containing carbanions $\left(\mathrm{C}_{x} \mathrm{~N}_{y}{ }^{-}\right)$. 
Carbon chain anions $\left(C_{x}^{-}\right)$. Pure carbon chain anions, with $\mathrm{x}=1-11$, can be formed in the ion production region using a cold cathode dc discharge; a graphite rod is raised to several kilovolts relative to the flow tube. Helium and argon are used as carrier gases, and the discharge is stabilized by water-cooling. An anion with a specific mass-to-charge ratio is selected with the SIFT quadrupole, and injected into the reaction flow tube for study. None of the carbon chain anions react with molecular hydrogen, but they react efficiently with hydrogen atoms by associative detachment. The larger carbanions also show simple addition to form the hydrogenated carbon chain anions. Typical rate constants are about $7 \times 10^{-10} \mathrm{~cm}^{3} \mathrm{~s}^{-1}$ (Barckholtz et al. 2001).

Reactions of the carbon chain anions with atomic oxygen occur rapidly by associative detachment and by the loss of a single carbon atom to form carbon monoxide and an anion with one fewer carbon atom. Reactions of carbon chain anions with nitrogen atoms are somewhat slower $\left(\sim 2 \times 10^{-10} \mathrm{~cm}^{3} \mathrm{~s}^{-1}\right)$, but show a rich variety of pathways that involve formation of carbon-nitrogen bonds (Eichelberger et al. 2007; Yang et al. 2010a).

Hydrogenated carbon chain anions $\left(C_{x} H^{-}\right)$. These anions do not react with molecular hydrogen but undergo rapid associative detachment reactions with hydrogen atoms (Barckholtz et al. 2001). With oxygen atoms, these anions show associative detachment, transfer of carbon to form carbon monoxide, as well as replacement of hydrogen by oxygen . The reactions with nitrogen atoms show pathways similar to those for the pure carbon chain anions, but the reactions are extremely slow; this lack of reactivity is attributed to problems of spin conservation for the reaction of the singlet anions with the quartet nitrogen atoms (Eichelberger et al. 2007).

Organic anions $\left(H_{w} C_{x} N_{y} O_{z}^{-}\right)$. We have explored the reactivity of several organic anions formed by deprotonation of the neutral parents, including methyl cyanide, ethyl cyanide, iso-propyl cyanide, acetaldehyde, acetone, ethyl acetate, methanol, acetic acid and glycine (Snow et al. 2009; Yang et al. 2010b). Reactions of these anions with hydrogen atoms occur by associative detachment, and there is a direct correlation between reactivity and the reaction exothermicity. The low reactivity of the acetate and glycine anions reflects the high stability of these delocalized anions.

In all cases, the reactions of these organic anions with oxygen atoms have larger rate constants relative to the corresponding reactions with nitrogen atoms. Our computational studies indicate that spin-allowed reactions dominate the O-atom reactions, whereas slow spin-forbidden pathways occur for the N-atom reactions (Yang et al. 2010c).

Hydride anion $\left(H^{-}\right)$. The reactions of hydride ion with a series of neutral reagents occur by a wide array of mechanisms (Martinez et al. 2010). Efficient proton abstraction processes are observed for hydride anion reacting with acetylene, water, methyl cyanide, methanol, acetone, and acetaldehyde. Facile associative detachment is observed for molecular oxygen, whereas extremely slow associative detachment is observed for carbon monoxide. Reaction of hydride with nitrous oxide forms the hydroxide ion, while reaction with carbon dioxide occurs by addition to produce formate anion. Reaction of hydride with methyl chloride occurs by nucleophilic substitution $\left(\mathrm{S}_{N} 2\right)$, whereas reaction with tert-butyl chloride proceeds by elimination (E2); both of these processes generate chloride ion as the product. Finally, reactions of hydride ion with diethyl ether, benzene, and molecular deuterium were immeasurably slow.

Nitrogen-containing carbanions $\left(C_{x} N_{y}^{-}\right)$. These species are generated in the ion production region with the cold cathode discharge source, using the graphite rod in the presence of molecular nitrogen (Yang et al. 2011).

A. Ions containing one nitrogen atom. We have mass-selected and studied anions containing a single nitrogen atom, and from one to six carbon atoms, as shown in Figure 2. 


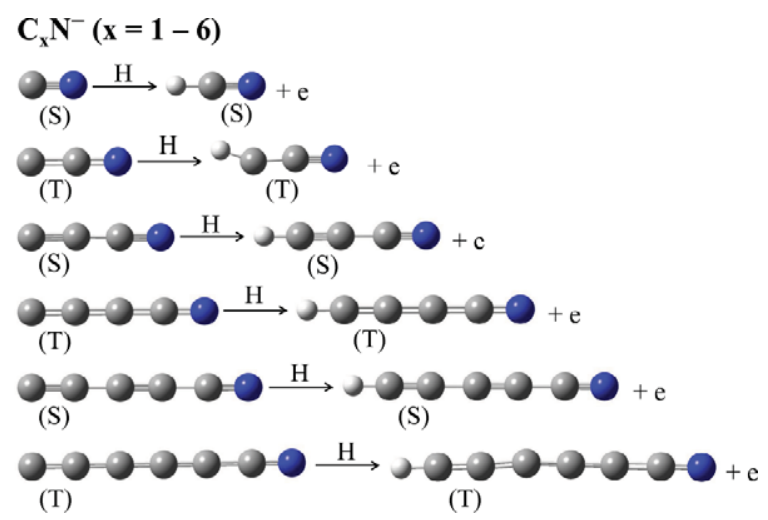

Figure 2. Associative Detachment Reactions of $\mathrm{C}_{x} \mathrm{~N}^{-}$. The singlet ground state is designated by $\mathrm{S}$, and the triplet ground state is designated by $\mathrm{T}$.

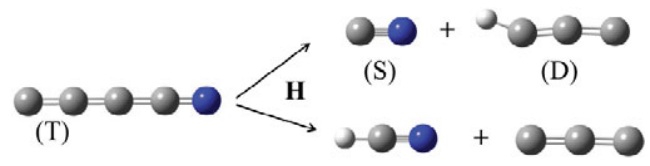

(S)

(D)

Figure 3. Reaction of $\mathrm{C}_{4} \mathrm{~N}^{-}$with Hydrogen Atom to Form Ionic Products. S, D, and T designate singlet, doublet, and triplet, respectively.

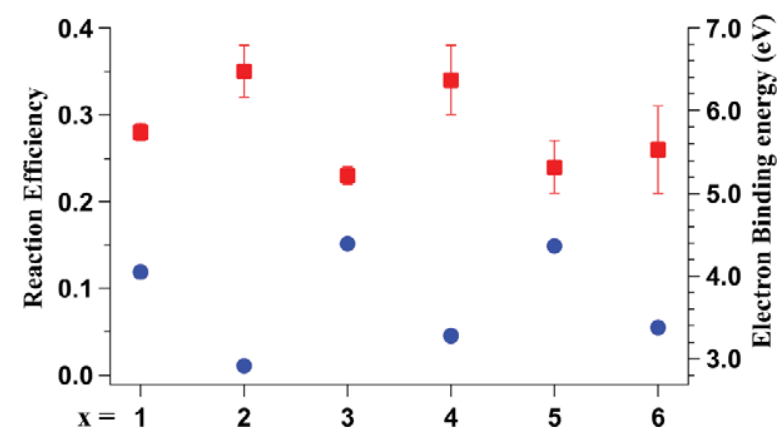

Figure 4. Reaction Efficiency (squares) for $\mathrm{C}_{x} \mathrm{~N}^{-}+\mathrm{H}(\mathrm{x}=1-6)$ and the Electron Binding Energy of the Anions (circles).

The $\mathrm{S}$ and $\mathrm{T}$ designations indicate the singlet or triplet nature of the ground electronic state, as determined by our computations. The anions are unreactive with molecular hydrogen, but react readily with atomic hydrogen by associative detachment, to form for example, HCN and an electron. The larger anions react in a similar manner. Some of the anions, for example $\mathrm{C}_{4} \mathrm{~N}^{-}$, also give ionic products; as shown in Figure 3, reaction with $\mathrm{H}$-atom forms $\mathrm{CN}^{-}$and $\mathrm{HC}_{3}$, or products in which there is a proton transfer to form $\mathrm{HCN}$ and $\mathrm{C}_{3}{ }^{-}$. Although these reactions are relatively rapid, there is a slight alternation in reactivity. If reaction efficiency is plotted versus the number of carbon atoms (Figure 4), the ions with an even number of carbon atoms have slightly higher reactivity as indicated by the squares, but have lower electron binding energies as indicated by the circles.

B. Ions containing two nitrogen atoms. We have studied the reactions of the anions $\mathrm{C}_{x} \mathrm{~N}_{2}{ }^{-}$, where $\mathrm{x}=1,3,4$, and 5 . These doublet negative ions react readily with $\mathrm{H}$-atom by associative detachment; $\mathrm{C}_{4} \mathrm{~N}_{2}{ }^{-}$and $\mathrm{C}_{5} \mathrm{~N}_{2}{ }^{-}$also give ionic products. 


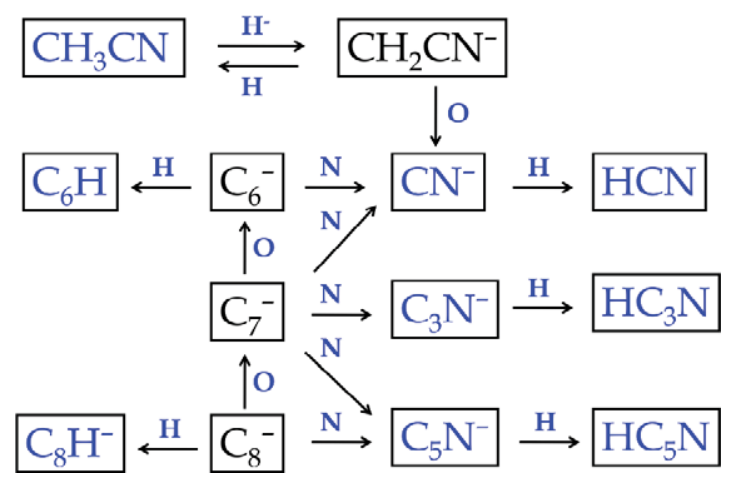

Figure 5. Partial Chemical Network for Interstellar Molecular Synthesis. This diagram illustrates some of the reactions studied in our laboratory.

C. Ions containing three nitrogen atoms. The $\mathrm{C}_{2} \mathrm{~N}_{3}{ }^{-}$and $\mathrm{C}_{4} \mathrm{~N}_{3}{ }^{-}$anions show no measurable reactivity with hydrogen atoms. Our computational studies indicate that there are high energy transition states along the reaction surface, and only endothermic channels exist. Thus, these ions will exhibit high stability in the ISM, even in regions of high $\mathrm{H}$-atom density. The $\mathrm{C}_{4} \mathrm{~N}_{3}{ }^{-}$anion has a symmetrical structure $\left[\mathrm{C}(\mathrm{CN})_{3}{ }^{-}\right]$and therefore no dipole moment. In contrast, the $\mathrm{C}_{2} \mathrm{~N}_{3}{ }^{-}$anion is bent and polar; thus, this ion would be a good target for laboratory characterization of the rotational spectrum and for eventual astronomical detection.

\section{Summary}

Figure 5 shows nine molecules and anions that have been detected in the ISM, as well as four anions that have not been detected, but are likely present $\left(\mathrm{CH}_{2} \mathrm{CN}^{-}, \mathrm{C}_{6}{ }^{-}\right.$, $\left.\mathrm{C}_{7}{ }^{-}, \mathrm{C}_{8}{ }^{-}\right)$. This scheme provides a glimpse of how ion chemistry can contribute to interstellar synthesis and interconnect the observed species. The arrows represent some of the chemical reactions with hydrogen, nitrogen, and oxygen atoms described in this presentation. Clearly this ion-neutral chemistry can provide efficient avenues for the generation and interconversion of these molecular ions and neutrals.

\section{Future Directions}

Many additional studies are desirable. In particular, we plan to quantify the product ratios for many of the reactions; these studies require that we account for mass discrimination, secondary reactions, and the loss of ion signal due to associative detachment. In addition, we plan to study the reactions of the nitrogen-containing carbanions with nitrogen and oxygen atoms.

Finally, we propose to extend our studies to larger ionic species, including polycyclic aromatic hydrocarbons and biomolecules. In preliminary studies, we have successfully generated deprotonated benzene $\left(\mathrm{C}_{6} \mathrm{H}_{5}{ }^{-}\right)$, naphthalene $\left(\mathrm{C}_{10} \mathrm{H}_{7}{ }^{-}\right)$, and anthracene $\left(\mathrm{C}_{14} \mathrm{H}_{9}{ }^{-}\right)$, and explored their ion chemistry. These singlet anions have moderate electron binding energies, and may be readily formed in molecular clouds; therefore, these anions should be included in the modeling of cloud chemistry. In contrast, the doublet molecular anions benzene $\left(\mathrm{C}_{6} \mathrm{H}_{6}{ }^{-}\right)$, naphthalene $\left(\mathrm{C}_{10} \mathrm{H}_{8}{ }^{-}\right)$, and anthracene $\left(\mathrm{C}_{14} \mathrm{H}_{10}{ }^{-}\right)$, formed by electron attachment to the parent molecules, are more weakly bound. 
In order to extend these studies to larger compounds of lower volatitily, we are currently incorporating Laser Induced Acoustic Desorption (LIAD) and Electrospray Ionization (ESI) as new ion sources for our FA-SIFT. We hope that these modifications will enable the study of a broad range of chemical systems.

\section{Acknowledgements}

NASA and the National Science Foundation are gratefully acknowledged for their financial support of this research. I am grateful to my collaborators for their invaluable contributions to this work: Ted Snow, Zhibo Yang, Nicholas Demarais, Callie Cole, Nadine Wehres, Oscar Martinez, Nick Betts, Brian Eichelberger, and Cindy Barckholtz. I thank Zhibo Yang for help in preparing the figures, and Nicholas Demarais and Callie Cole for creating the Latex version of this manuscript.

\section{References}

Agúndez, M., Cernicharo, J., Guélin, M., Kahane, C., Roueff, E., Klos, J., Aoiz, F. J., Lique, F., Marcelino, N., Goicoechea, J. R., González García, M., Gottlieb, C. A., McCarthy, M. C., \& Thaddeus, P. 2010, A\& $A, 517$, L2

Barckholtz, C., Snow, T. P., \& Bierbaum, V. M. 2001, ApJ, 547, L171

Brünken, S., Gupta, H., Gottlieb, C. A., McCarthy, M. C., \& Thaddeus, P. 2007, ApJ, 664, L43

Cernicharo, J., Guélin, M., Agúndez, M., Kawaguchi, K., McCarthy, M., \& Thaddeus, P. 2007, $A \mathscr{E} A, 467, \mathrm{~L} 37$

Cernicharo, J., Guélin, M., Agúndez, M., McCarthy, M. C., \& Thaddeus, P. 2008, ApJ, 688, L83

Douglas, A. E. \& Herzberg, G. 1941, ApJ, 94, 381

Eichelberger, B., Snow, T. P., Barckholtz, C., \& Bierbaum, V. M. 2007, ApJ, 667, 1283

Martinez Jr., O., Yang, Z., Demarais, N. J., Snow, T. P., \& Bierbaum, V. M. 2010, ApJ, 720, 173

McCarthy, M. C., Gottlieb, C. A., \& Gupta, H., Thaddeus. P. 2006, ApJ, 652, L141

Remijan, A. J., Hollis, J. M., Lovas, F. J., Cordiner, M. A., Millar, T. J., Markwick-Kemper, A. J., \& Jewell, P. R. 2007, ApJ, 664, L47

Snow T. P., Bierbaum, V. M. 2008, Annu. Rev. Anal. Chem., 1, 229

Snow, T. P., Stepanovic, M., Betts, N. B., Eichelberger, B. R., Martinez Jr., O., \& Bierbaum, V. M. 2009, Astrobiol., 9, 1001

Thaddeus, P., Gottlieb, C. A., Gupta, H., Brünken, S., McCarthy, M. C., Agúndez, M., Guélin, M., \& Cernicharo, J. 2007, ApJ, 677, 1132

Van Doren, J. M., Barlow, S. E., DePuy, C. H., \& Bierbaum, V. M. 1987, Int. J. Mass Spectrom. Ion Proc., 81, 85

Yang, Z., Snow, T. P., \& Bierbaum, V. M. 2010a, Phys. Chem. Chem. Phys., 12, 13091

Yang, Z., Eichelberger, B., Carpenter, M. Y., Martinez Jr., O., Snow, T. P., \& Bierbaum, V. M. 2010b, ApJ, 723, 1325

Yang, Z., Eichelberger, B., Martinez Jr., O., Stepanovic, M., Snow, T. P., \& Bierbaum, V. M. 2010c, J. Am. Chem. Soc., 132, 5812

Yang, Z., Cole, C. A., Martinez Jr., O., Carpenter, M. Y., Snow, T. P., \& Bierbaum, V. M., ApJ, in press 2011.

\section{Discussion}

HERBST: Can you study attachment reactions?

BIERBAUM: Yes, in principle, electron attachment can be studied in flow tube instruments by incorporating a Langmuir Probe. However, we have not pursued studies in this area.

SмiтH: Can you confirm that you can observe and measure the reaction rates of $\mathrm{C}_{3}{ }^{-}$? I had the impression that it was missing from some of your slides. 
BIERBAUM: Although $\mathrm{C}_{3}{ }^{-}(\mathrm{m} / \mathrm{z} 36)$ is generated in the graphite discharge source, there are chloride ion impurities present at $\mathrm{m} / \mathrm{z} 35$ and 37 . Therefore, it is difficult to mass select and inject a clean signal of $\mathrm{C}_{3}{ }^{-}$for studies of the chemistry.

SCHLEmmer: What are the routes to form $\mathrm{C}_{x}$ - in space? (Reference to your chemical network diagram where such a route is missing.) Please comment.

BiERBAUm: The carbon chain anions are most likely formed in space by electron attachment to neutral carbon chains; radiative electron attachment to the larger carbon chains should be facile. The chemical network diagram (Figure 5) includes only reactions that we have studied, and represents only a sampling of our work.

Hama: Could you please give us a comment on how you confirmed the structures of the reactant and products?

Bierbaum: Many ionic structures are possible for a given $\mathrm{m} / \mathrm{z}$ value. The reactant and product ions shown in the slides are the most stable isomers as determined by our computations. Structures could be probed by collision-induced dissociation experiments, by mobility studies, or possibly by spectroscopic experiments. 